\title{
Untersuchungen an einem 15-MV-Betatron
}

\author{
Von Rudolf Kollath und Gerhard Schumann* \\ (Z. Naturforschg. 2 a, 634-642 [1947]; eingegangen am 22. Mai 1947)
}

\begin{abstract}
Aufbau und Wirkungsweise eines 15-MV-Betatrons werden kurz geschildert; im Anschluß daran wird über einige Untersuchungen berichtet, deren Ziel die Kenntnis der Eigenschaften und der Nachweis des richtigen Funktionierens des Gerätes war, um die Voraussetzungen für geplante Neukonstruktionen zu schaffen.
\end{abstract}

$\mathrm{U}$ nter einem Betatron wird bekanntlich ein Kreisbeschleuniger für Elektronen verstanden, bei dem die Beschleunigung durch das elektrische Wirbelfeld besorgt wird, das einen sich

zeitig von B. Tou s che k verschiedene theoretische Untersuchungen durchgeführt wurden.

Die Mehrzahl der Messungen wurde in Wrist, Mittelholstein, durchgeführt, wohin der Apparat

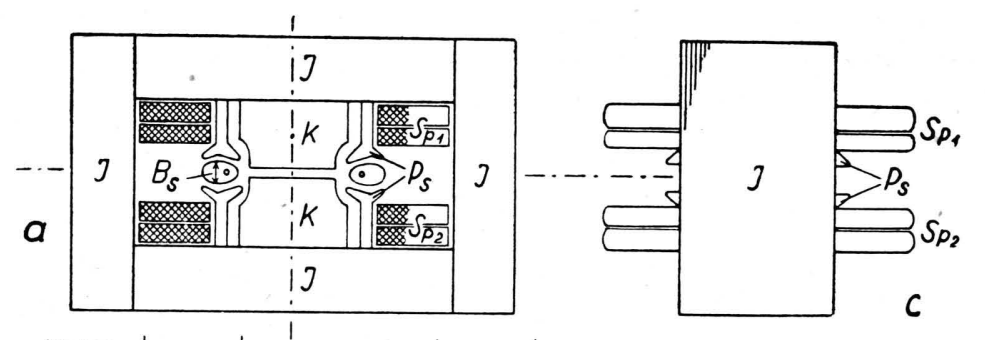

$b$

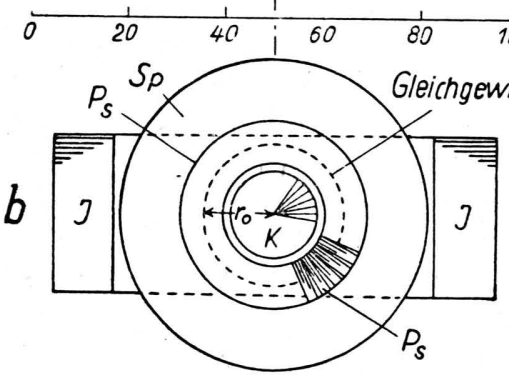
gegen Kriegsende verlagert wurde ${ }^{3}$. Die Arbeiten konnten nur bis Dezember 1945 fortgesetzt werden, so daß noch viele Fragen offen geblieben sind.

\section{Aufbau und Betriebsdaten des $15-M V-B e t a t r o n s$}

Abb. 1 gibt eine Übersicht über die Gesamtanordnung. Zwei Doppelspulen $\mathrm{Sp}_{\mathbf{1}}$ und $\mathrm{Sp}_{2}$ erzeugen im Kern $\mathrm{K}$, der mit einem Luftspalt versehen ist, den induzierenden Fluß und gleichzeitig das magnetische Steuerfeld $B_{\mathbf{s}}$ zwischen den ringförmigen Polschuhen $\mathrm{P}_{\mathbf{s}}$, die einen mittleren Abstand von $6 \mathrm{~cm}$ haben. In der Mitte zwischen den Steuerpolen ist durch einen Punkt der „Gleichgewichtskreis“ bezeichnet, auf den der ringförmige Elektronenstrahl sich im Verlauf der Beschleunigung zusammenzieht. Zwischen den oberen und unteren Polschuhen liegt die toroidförmige

ändernden magnetischen Fluß umschließt. Die Elektronen werden dabei durch ein mit dem erwähnten magnetischen Fluß gekoppeltes magnetisches Führungsfeld auf einer Kreisbahn gehalten. Wegen der Grundlagen sei auf die Literatur verwiesen ${ }^{\mathbf{1}}$.

Ein solches Betatron zur Erzeugung von Elektronenstrahlen einer Energie von $15 \cdot 10^{6} \mathrm{eV}$ wurde Anfang 1944 in Hamburg von R. Wi d e rö e berechnet und in Gemeinschaft mit der Firma C. H. F. Müller, Hamburg, durchkonstruiert und aufgebaut ${ }^{2}$. Im Sommer 1944 wurde es von Wi d er öe und Kollath in Betrieb gesetzt, wobei gleich-

* (17 a) Neckarzimmern, Luttenbachtal.

1 R. W i d e r ö e, Diss. T. H. Aachen 1927; Arch. Elektrotechn. 21, 387 [1928]; St e e n be c k, DRP 656378 u. 698867 [1937]; D. K e r st. Physic. Rev. 60, 47 [1941]; K e r s t u. Se r b e r, Physic. Rev. 60, 53 [1941] u. a.m.
Vakuumröhre, in welcher die Elektronen beschleunigt werden. Ein Eisenjoch $J$ schließt den magnetischen Kraftfluß des Kerns und der Steuerpole. Der Kern ist aus radial geschichtetem Trafoblech aufgebaut, die Polschuhe ebenfalls, wobei die Bleche zu Paketen zusammengefaßt sind (Abb.1b). Das Joch besteht aus 4 Teilen, die mit Hilfe von U-Eisen gegeneinander verschraubt sind. Über dem Apparat befindet sich ein Flaschenzug, mit dem nach Lösung der Verschraubungen der obere Teil des Joches mit oberem Kern und oberem Steuerpol nach oben weggehoben werden kann, um die Ringröhre einsetzen und an ihr hantieren zu können. Der gesamte Apparat ist auf einem Tisch aufgebaut. Eine Anschauung von

2 Wir danken den HHrn. der Fa. C. II. F. M ü 11 e r für jederzeitige tatkräftige Unterstützung und ihr Interesse an dem Fortgang der Arbeit.

3 Wir möchten auch an dieser Stelle Hrn. Fabrikbesitzer Richard S e i f e r t, Hamburg, dafür danken daß er uns stets bereitwilligst seine Hilfe zur Verfügung gestellt hat. 
seinem Aussehen und den Größenverhältnissen soll Abb. 2 vermitteln, die zahlenmäßigen Daten der Anordnung sind in Tab. 1 zusammengestellt.

Beim Betrieb werden die

Erregerspulen mit Wechselstrom aus dem normalen Netz beschickt (Abb.3). Die Elektronen werden aus einer Glühkathode bei der Phase $0^{\circ}$ des Magnetfeldes in der Röhre gestartet. Die Steuerpole sind in ihren dem Joch benachbarten Teilen so dimensioniert, daß sie sich bei einem Phasenwinkel von etwa $60^{\circ}$ bis $70^{\circ}$ sättigen. Das Feld zwischen den Steuerpolen bleibt dann hinter dem Anstieg des Induktionsfeldes zurück, wodurch sich der Elektronenkreis ausdehnt und dabei schließlich auf die Antikathode auftrifft.

Die entstehenden Röntgenstrahlen treten mit einer Energie bis zu $15 \mathrm{MeV}$ aus der Röhre nach außen.

Beim Betatron muß für den projektierten Radius

\begin{tabular}{l}
\hline A b m e s s u g e \\
Jochbreite $92 \mathrm{~cm}$ \\
Jochhöhe $56 \mathrm{~cm}$ \\
Jochquerschnitt $13 \times 32 \mathrm{~cm}$ \\
Kernradius $11 \mathrm{~cm}$, in der Mitte auf $9 \mathrm{~cm}$ verjüngt \\
Luftspalt im Kern $2 \mathrm{~cm}$ \\
Luftspalt zwischen den Steuerpolen $6 \mathrm{~cm}$ (Mittel) \\
Radius des Gleichgewichtskreises $14 \mathrm{~cm}$ \\
Gesamtgewicht $1,2 \mathrm{t}$, davon $1 \mathrm{t}$ Eisen und $0,2 \mathrm{t} \mathrm{Kupfer.}$
\end{tabular}

M a g n etfeld und Elektronenkreis maximale Induktion im Kern ca. $15000 \mathrm{Gauß}$ maximale Induktion im Gleichgewichtskreis $3700 \mathrm{Gauß}$ Exponent des Feldabfalls $n=1 / 2$

Energiegewinn/Umlauf $14,3 \mathrm{eV}$

Umlaufzahl ca. $1,2 \cdot 10^{6}$

Endenergie ca. $15 \mathrm{MeV}$

Einschußspannung $7,5 \mathrm{kV}$

mittlere Einschußstromstärke.100 $\mu \mathrm{A}$

Einschußzeit 2 bis $5 \cdot 10^{-4}$ sec

Betriebsvakuum 5 bis $8 \cdot 10-6$ Torr

Dicke der Antikathode $2.5 \mathrm{~mm}$

Maximalintensität $1,2 \mathrm{r} / \mathrm{min}$.

Elektrische Daten

Erregerspule $280 \mathrm{Wdg}$. $3,5 \times 3,5 \mathrm{~mm}^{2}$, 4-fach parallel Erregerspannung $5 \mathrm{kV}$

Erregerstrom $58 \mathrm{~A}$ eff.

Blindleistung $280 \mathrm{kVA}$

Kondensatoren $33 \mu \mathrm{F}$

Bleche für Kern und Joch $0,35 \mathrm{~mm}$ Trafoblech $1,1 \mathrm{~W} / \mathrm{kg}$ Verluste im Betatron 2,5 kW

Kühlung keine.

Tab. 1. Daten des 15-MV-Betatrons. des Gleichgewichtskreises bekanntlich das Feld zwischen den Steuerpolen- an der Stelle des Gleichgewichtskreises halb so groß sein wie der mittlere induzierende Fluß innerhalb dieses Kreises. Dies wird durch ein geeignetes Verhältnis der Luftspalte im Kern und zwischen den Steuerpolen erreicht, wobei nachträglich kleine Korrekturen möglich sind, da der obere Teil des Jochs mit obeTombak- rem Kern und rohr oberem Steuer pol in seiner Ölpumpe Lage innerhalb zen mittels Stellschrauben veränderlich ist. Ferner muß das Feld zwischen den Steuerpolen einen bestimmten radialen Feldabfall nach außen hin besitzen, damit die Elektronenbahnen innerhalb des Bereichs zwischen den Steuerpolen stabil sind. Dies wird durch geeignete Formgebung der Steuerpolschuhe erreicht; der Exponent des Feldabfalls zwischen den Steuerpolen ist $n=1 / 2$. Die Polschuhform sowie die $B$-Kurve (Feldverlauf) nebst der $r \cdot B$-Kurve (Verlauf der Stabilisierungskraft) ist in Abb. 4 genauer wiedergegeben.

Die aus Glas gefertigte Ringröhre (Abb.5) besitzt zwei Ansätze mit Planschliffen, von denen der eine als Pumpstutzen, der andere zur Einbringung der Glühkathode dient. Die Ringröhre ist mit einem schwach leitenden Innenbelag ausgekleidet, der in den meisten Fällen aus Graphit (Aquadag), bei einigen Versuchsröhren aus Silber besteht und geerdet

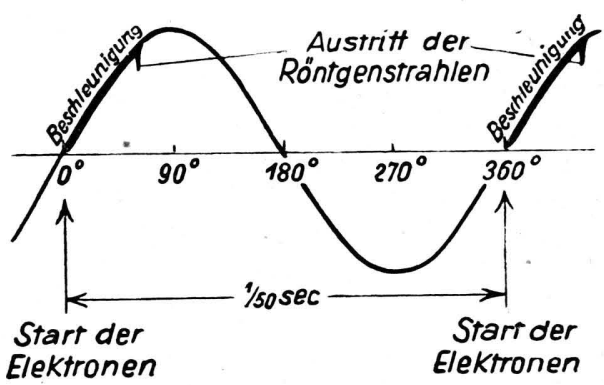

Abb. 3. Stromkurve für die Erregerspulen mit Betriebszeichnung.

ist. Abb. 6 zeigt die zuletzt benutzte Kathodenanordnung: Eine Oxydkathode von etwa $1 \times 10 \mathrm{~mm}^{2}$ emittierender Fläche ist von einem etwa auf gleichem Potential befindlichen Wehnelt-Zylinder in geringem Abstand umgeben; die Anodenplatten sind voneinander isoliert, so daß ihnen verschiedenes Potential 
gegeneinander und gegen die Kathode erteilt werden kann. Dies bietet neben der-eigentlichen Beschleunigung der Elektronen die Möglichkeit, den Elektronenstrahl in azimutaler Richtung abzulenken, ähnlich wie bei den Ablenkplatten eines Oszillographen. Dèr Wehnelt-Zylinder und die Anodenplatten bestehen aus $0,3 \mathrm{~mm}$ starkem Mo-Blech. Die vertikale Einjustierung des Elektronenstrahls wird mit Hilfe

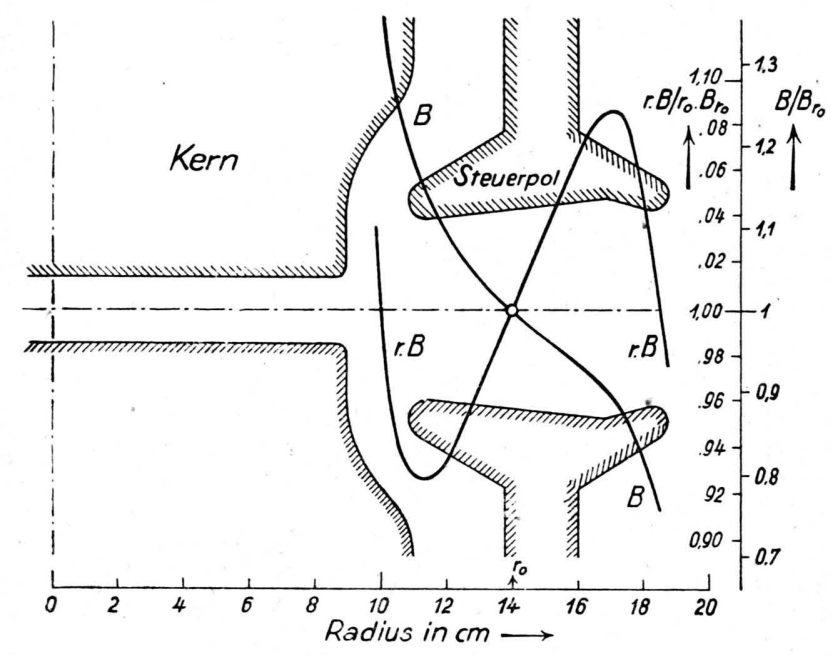

Abb. 4. Steuierpolschuhe mit Feldverlauf.

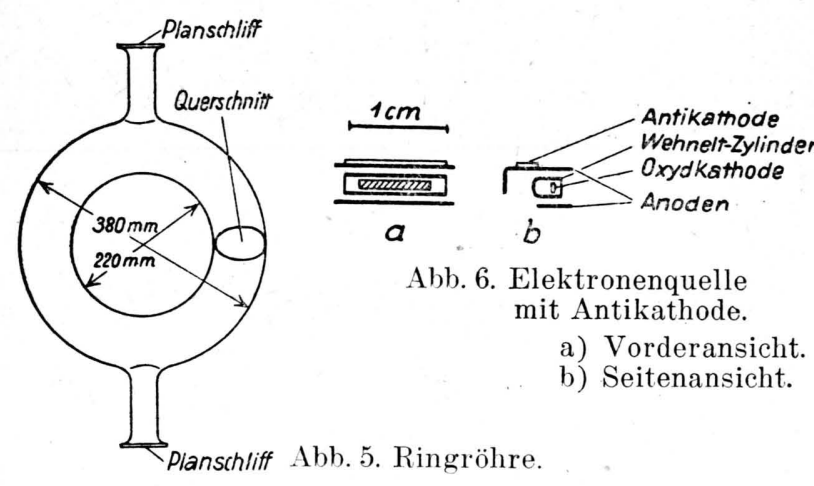

des oben erwähnten Schliffs vorgenommen, durch dessen Drehung während des Betriebes auf optimale Intensität eingestellt wird. An die Kathode und den Wehnelt-Zylinder wird im Betrieb eine Spannung von 6 bis $8 \mathrm{kV}$ kurzzeitig angelegt. Die Antikathode besteht aus W und ist an der Innenseite der Kathodenanordnung, also an dem inneren Anodenblech, befestigt.

Die Röhre wird mittels einer Öldiffusionspumpe mit Vorpumpen evakuiert. Zur Kontrolle des Vakuums während des Betriebes dient ein Philips-Vakumeter hinter der ölpumpe. Quantitative Druckmessungen können mit einem Ionisations-Manometer vorgenommen werden, das an dem Gluihkathodenansatz der Röhre angeschmolzen ist.
Der Erregerspulenkreis wird aus dem Drehstromnetz über einen Stufentrafo $380 / 5000 \mathrm{~V}$ betrieben, der bis zu $60 \mathrm{~A}$ eff. liefert. Die Blindleistung wird von Kondensatoren aufgenommen, von denen einer zwecks besserer Abgleichung des Schwingungskreises einige Unterteilungen besitzt. Für die Abführung der Wärmeverluste im Eisen des Magneten und in den Spulen wird eine besondere Kühlung durch Luft oder Wasser nicht benötigt.

Die Hochspannung für die Elektronenquelle wird einem Aggregat entnommen, bei dem eine Vakuumtriode mit Hilfe eines mit dem Primärkreis induktiv gekoppelten Sättigungstrafos in der Nähe des Nulldurchgangs des Magnetfeldes kurzzeitig geöffnet wird. Die Phasenlage der Impulssteuerung kann über einen Hilfstrafo geändert werden. Die Hilfsspannungen, z. B. an den Anoden, werden kleinen GleichspannungsAggregaten entnommen. Die Hochspannungsspitze an der Kathode wird auf einen Oszillographen geschrieben zusammen mit den Stößen eines Zählrohrs, das zum qualitativen Nachweis der entstehenden Röntgenstrahlen dient.

\section{Messungen zum Betrieb}

Da es sich bei diesem 15-MV-Betatron nur um eine erste Versuchsausführung handelte, mit der auf dem Wege zu größeren Geräten ${ }^{4}$ Betriebserfahrungen gesammelt werden sollten, war bei der Konstruktion das Hauptgewicht auf die Variationsmöglichkeit aller Versuchsbedingungen gelegt worden. Die Abhängigkeit der Funktion des Apparates von Form und Lage der Kathode, vom Potential der Anodenbleche, von der Lage der Polschuhe zueinander usw. wurde ausführlich studiert. Erst als nach solchen Untersuchungen eine befriedigende Intensität und Konstanz der erzeugten Röntgenstrahlung erreicht war, wurden auch an dieser selbst einige Messungen vorgenommen, die unter III besprochen werden. In Abschnitt II werden die hauptsächlichsten Ergebnisse der Messungen bei verschiedenen Betriebsbedingungen wiedergegeben, da einige der hier gemachten Erfahrungen vielleicht für spätere Konstruktionen von Wert sein können.

Elektronenquelle. Im Anfang wurde mit Glühkathoden gearbeitet, die aus einem Wolframdraht oder einer Wolframwendel bestanden. Diese Kathoden zeigten iedoch durchweg einen starken zeitlichen Intensitätsabfall, der mit Erhöhung der Gesamtintensität wuchs. Diese Erscheinung trat sowohl im Dauerbetrieb als auch bei regelmäßig unterbrochenem Betrieb auf, eine Erholung trat

${ }^{4}$ Ein 200-MV-Gerät war Ende 1944 in Konstruktion, außerdem war der Bau eines 30-MV-Geräts für medizinische $\mathrm{Zwecke}$ geplant. 
erst nach längeren Pausen von der Größenordnung einer Stunde ein. Die verschiedenen Untersuchungen zu dieser Erscheinung deuteten darauf hin, daß es sich um ein mechanisches Verziehen der Kathodenteile gegeneinander handelte, das durch die verschiedene Erwärmung der Teile und ihrer Zuleitungen beim Betrieb der Kathode bedingt war. Mit der Benutzung von Oxydkathoden mit ihren erheblich niedrigeren Temperaturen hörte diese Störung auf, und es ergaben sich auch bei mehrstündigem Betrieb reproduzierbare Intensitäten ohne irgendeinen Gang nach oben oder unten. Da sich im Betatron stets nur die Endintensität beobachten ließ, wurden die Kathoden hinsichtlich ihrer Emissionseigenschaften in einer besonderen Prüfröhre mit Leuchtschirm untersucht, wobei im übrigen unter den gleichen Betriebsbedingungen gearbeitet wurde wie in der Hauptapparatur.

Da neuere theoretische Untersuchungen über den zulässigen Aziputalwinkelbereich, innerhalb dessen die Elektronen noch von dem Steuerfeld „eingefangen“werden, nur Winkel von der Größenordnung $1 / 2^{\circ}$ ergeben haben, ist $\mathrm{zu}$ schließen, daß die hier verwendeten Elektronenquellen gerade in dieser Hinsicht noch erheblicher Verbesserung bedürfen und daß solche Verbesserungen zur Verminderung der Störung durch ,falsche“ Elektronen ${ }^{5}$ und damit zu einer Intensitätssteigerung führen werden.

Potentiale an den Hilfselektroden. Von erheblichem Einfluß auf die Intensität zeigte sich das an die Anodenbleche angelegte Potential. Ein Beispiel für diese Abhängigkeit ist in Abb. 7 wiedergegeben, in der als Abszisse das Potential der einen Anodenplatte (die andere Anodenplatte sowie der leitende Innenbelag der Röhre waren geerdet) und als Ordinate die Intensität aufgetragen ist.

Die Wirkung der Anodenaufladung wurde ursprünglich als reine Ablenkwirkung gedeutet, d.h. es wurde als wesentlich angesehen das Feld, das sich zwischen der aufgeladenen und der geerdeten Anode ausbildet und das auch zweifellos den aus

5 Als „falsche“ Elektronen werden solche bezeichnet, die auf Grund ihrer Einschußbedingungen nur einige Male in der Ringröhre umlaufen und dann wieder herausfallen. Sie verhindern durch die von ihnen erzeugte Raumladung während des Einschießvorganges die Füllung der Röhre mit solchen Elektronen, die den Beschleunigungsvorgang bis zum Ende mitmachen. der Kathode austretenden Elektronenstrahl abzulenken imstande ist, wie sich u. a. in der Kathodenprüfröhre gezeigt hat, wo sich allerdings die $\mathrm{Ab}$ lenkwinkel als ziemlich klein herausstellten. Später erhielten wir aber experimentelle Befunde, die eine Anderung dieser Auffassung erforderlich machten. Es ergab sich, daß in jedem Fall die an die innere Anodenplatte angelegte Spannung den entscheidenđen Einfluß auf die Intensität ausübte und daß die Optima bei relativ hohen positiven Spannungen lagen. Es war nicht möglich, auch nur die Größenordnung der so erhaltenen Intensitäten zu bekommen, wenn man die innere Platte

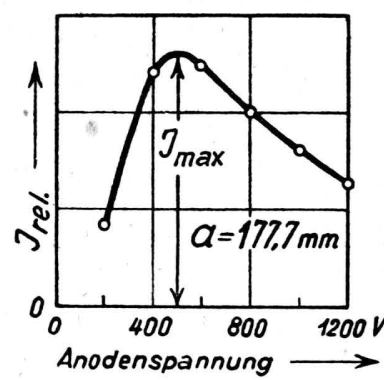

Abb. 7.

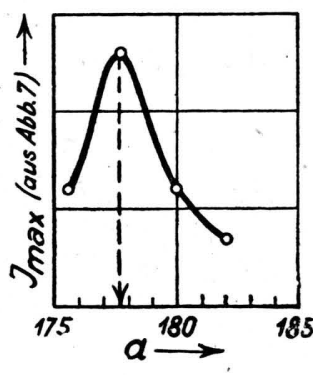

Abb. 8
Abb. 7. Intensität $I_{\text {rel }}$ der Röntgenstrahlen als Funktion der Anodenspannung. a Mittelpunktsabstand der Kathode von der Vertikalachse des Betatrons.

Abb. 8. Maximale Intensität $I_{\max }$ der Röntgenstrahlen (nach Abb. 7) als Funktion der Kathodenlage (Mittelpunktsabstand $a$ der Kathode, Parameter der Abb. 7).

erdete und die äußere auf entsprechendes negatives Potential brachte. Vielmehr zeigte sich, daß man an die äußere Platte wiederum eine positive Spannung legen mußté, um ein Intensitätsoptimum zu erreichen, das allerdings hinter dem absoluten Optimum bei Erdung der äußeren und positiver Spannung an der inneren Platte weit zurückblieb. Daraus ergibt sich, daß offenbar die ablenkende Wirkung, die sich ja bei den beiden beschriebenen Anordnungen in entgegengesetztem Sinne hätte geltend machen müssen, für die Endintensität bei den verwendeten Elektronenquellen eine untergeordnete Rolle spielt. Sie wird überdeckt von einem anderen Effekt, der vermutlich auf das Feld zurückzuführen ist, das sich zwischen der auf positivem Potential befindlichen inneren Anodenplatte und dem geerdeten Belag an der Innenwand der Röhre ausbildet und anscheinend einen wesentlich besseren Schutz vor Störungen der bereits umlaufenden Elektronen durch 
die Elektronenquelle bietet als die umgekehrte Anordnung mit geerdeter innerer und aufgeladener äußerer Anodenplatte.

Die Kurve in Abb. 7 gilt für eine bestimmte Lage der Elektronenquelle zum Gleichgewichtskreis. Da der Gleichgewichtskreis als Bezugswert unzweckmäßig ist, wurde die Lage der Elektronenquelle durch ihren Mittelpunktsabstand $a, \mathrm{~d}$. h. durch ihre Entfernung von der Vertikalachse des Betatrons, festgelegt. Ändert man den Mittelpunktsabstand, so ergibt sich, wie zu erwarten, eine andere optimale Anodenspannung und ein anderer optimaler Intensitätswert $I_{\max }$. Durch systematische Variation kann man die günstigste Kathodenlage ermitfeln. Dies wird in Abb. 8 an einem Meßbeispiel erläutert. Hier ist als Abszisse der Mittelpunktsabstand $a$ der Kathode (Parameter aus Abb. 7) aufgetragen und als Ordinate der zugehörige Maximalwert der Intensität $I_{\max }$ aus Abb. 7.

P o l a bstand. Messungen der eben beschriebenen Art wurden für verschiedene Polabstände durchgeführt. Der Polabstand konnte mit Hilfe der erwähnten Stellschrauben um Zehntel-mm geändert werden. Gemessen wurde der Abstand der Steuerpole mittels eines Innentasters an vier markierten Stellen, die auf dem Umfang um je $90^{\circ}$ gegeneinander verschoben waren. Bei Anderung des Polabstandes wird wegen der festen Verbindung zwischen Kern und Steuerpolen gleichzeitig der Luftspalt im Kern und der zwischen den

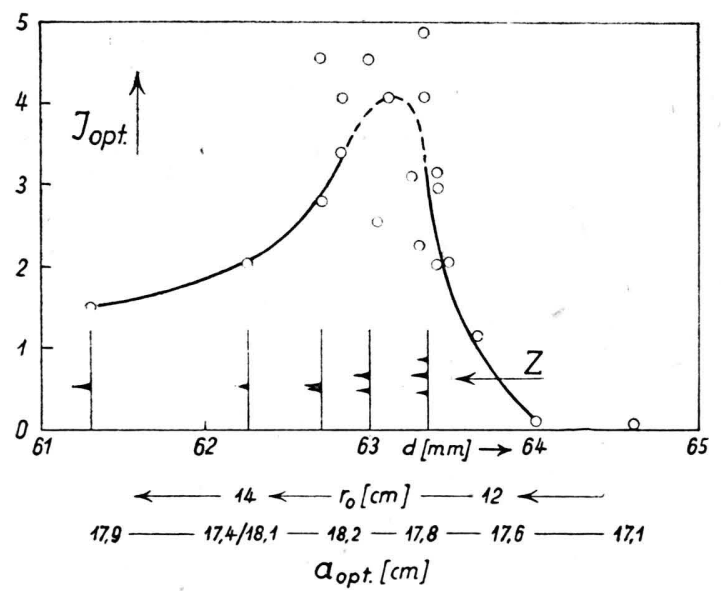

Abb. 9. Optimale Intensität $I_{\text {opt der Röntgenstrahlen }}$ (nach Abb. 8) als Funktion des Abstandes $d$ zwischen den Steuerpolen. $r_{0}$ Radius des Gleichgewichtskreisos in cm, $a_{\text {opt }}$ optimaler Mittelpunktsabstand der Glühkathode, $Z$ Aussehen der Zählrohrimpulse im Oszillogramm (schematisch).
Steuerpolen um den gleichen Absolutbetrag geändert, was sich aber auf den Kernabstand relativ stärker auswirkt, da dieser nur ein Drittel des Abstandes der Steuerpole beträgt. Aus den vier gemessenen Abstandswerten wurde ein mittlerer Polabstand ermittelt und für diesen die zugehörige Maximalintensität nach dem Vorgang von Abb. 7 und 8 bestimmt. Abb. 9 zeigt das Resultat dieser Messungen: Während bei einer Vergrößerung des mittleren Polabstandes über 63,3 mm ein schneller Abfall eintritt (Erhöhung des Steuerpolabstandes um ca. 1\% bewirkt Absinken der Intensität auf 1\%), fällt bei Verkleinerung des Polabstandes die Intensität nur langsam ab (Verkleinerung des Steuerpolabstandes um 1\% läßt die Intensität nur um die Hälfte absinken). Wenn auch die Maximalwerte ziemlich starke Schwankungen aufweisen, so gibt Abb. 9 doch ein gutes Bild der gesuchten Abhängigkeit im allgemeinen.

Der Intensitätsverlauf in Abb. 9 läßt sich qualitativ folgendermaßen verstehen: Bei einem Radius des Gleichgewichtskreises von $r_{0}=14 \mathrm{~cm}$ befinden wir uns etwa bei der Abszisse 62 bis 62,5. Bei Vergrößerung des Polabstandes zieht sich der Gleichgewichtskreis zusammen, und zwar sinkt $r_{0}$ auf ca. $12 \mathrm{~cm}$, wenn wir zum Polabstand 63,5 bis 64 fortschreiten. In diesem Fall fällt die Intensität steil ab. Wenn $r_{0}$ nämlich so klein geworden ist, hat der Gleichgewichtskreis in der benutzten Röhre keinen Platz mehr, die Elektronen stoßen bei ihren Umläufen (Schwingungen) an der gegenüberliegenden Innenwand der Röhre an. Dieser Effekt wird noch dadurch verstärkt, daß die Elektronenquelle, die ja hier mit der Röhre fest verbunden ist, bei Zusammenziehung des Gleichgewichtskreises auch nach innen rücken muß, um nicht weit außerhalb des Stabilitätsgebietes zu geraten; ihr optimaler Mittelpunktabstand wird mit Vergrößerung des Polabstandes entsprechend der Schrumpfung des Gleichgewichtskreises kleiner, wie die in Abb. 9 angeschriebenen Werte zeigen.

Bei Verkleinerung des Polabstandes, also Vergrößerung des Gleichgewichtskreises, erfolgt ein langsamer Abfall der Intensität, weil wir mit der Elektronenquelle relativ dem sich ausdehnenden Gleichgewichtskreis näherkommen und damit die Elektronenquelle immer mehr Elektronen wegfängt. Die Intensität 0 wird dabei erst erreicht, wenn der Gleichgewichtskreis sich so weit ausgedehnt hat, daß die Elektronenquelle praktisch 
auf ihn selbst zu liegen kommt; dieser Polabstand liegt weit außerhalb von Abb.9. Zwischen dem Intensitätsabfall nach kleineren und nach größeren Polabständen liegt ein Gebiet optimalen Abstandes. Die starken Intensitätsschwankungen in diesem Bereich sind wohl so zu deuten, daß hier der in der Ringröhre umlaufende Elektronenstrahl besonders im Gebiet beginnender Sättigung labil wird. Darauf deutet auch eine andere Beobachtung hin: Der Zeitpunkt der Entstehung der Röntgenstrahlen (Zählrohrspitze im Oszillogramm) zeigt in diesem optimalen Polabstandsgebiet eine Aufspaltung in mehrere Spitzen. (Das Aussehen der Zählrohrspitze für die verschiedenen Polabstände ist in Abb. 9 zwischen der Kurve und der Abszissenachse schematisch angedeutet.) Die Gründe für das Labilwerden des Elektronenstrahls bzw. die Aufspaltung der Zählrohrspitze konnten bisher allerdings noch nicht geklärt werden.

Polverkantung. Mit Hilfe der Stellschrauben konnte nicht nur der mittlere Polabstand variiert, sondern auch eine Verkantung der Polschuhe gegeneinander durchgeführt werden. Es zeigte sich bei Messungen dieser Art, daß optimale Intensität nicht bei paralleler Stellung der Polschuhe erreicht wurde, sondern bei einer gewissen, reproduzierbaren Verkantung der Polschuhe gegeneinander. Die in Aussicht genommene Ausmessung des Feldverlaufes zwischen den Polschuhen in dieser verkanteten Lage in Abhängigkeit vom Azimut sowie als Funktion des Radius in den verschiedenen Azimuten konnte nicht mehr durchgeführt werden. Es muß deshalb dahingestellt bleiben, ob diese Verkantung einem Ausgleich von Fertigungstoleranzen, also der Herstellung einer wirklichen Symmetrie der Anordnung, diente, oder ob durch sie eine geeignete Verformung des magnetischen Feldes in der Umgebung der Elektronenquelle hervorgerufen wird.

Die bei allen im Vorhergehenden besprochenen Versuchen gewonnenen Erfahrungen gestatteten es, auch nach Auseinandernehmen des Magneten und Entfernung der Ringröhre beim Zusammenbau von vornherein wieder auf die optimalen Bedingungen einzustellen, die durchaus reproduzierbar waren.

E ins chu $ß$ strom (Elektronenstrom von der Glühkathode in die Ringröhre). Bei den beschriebenen Untersuchungen wurden im allgemeinen nur mäßige Einschußströme verwendet, da die In- tensität für bequeme Messungen mit der Ionisationskammer ausreichte. Zum Schluß wurde untersucht, wieweit eine Steigerung des Einschußstromes eine entsprechende Steigerung der Intensität mit sich bringt. Hierbei ergab sich nach einem ziemlich linearen Anstieg mit dem Einschußstrom überraschenderweise schon bei mäßigen mittleren Einschußströmen ein Maximum; bei weiterer Steigerung des Einschußstromes fiel die Intensität wieder langsam ab (Abb. 10). Dies ist nur so zu verstehen, daß unter den vorhandenen Bedingungen die Füllung der Röhre erreicht ist und daß zusätzliches Einbringen von Elektronen in die Röhre nur noch die Anzahl der „falschen“ Elektronen vergrößert. Dieser Befund schließt nicht aus, daß man durch verschiedene Verbesserungen, z.B. der Elektronenquelle, noch Intensitätssteigerungen erzielen kann.

Vaku umbedingungen. Die so erreichte maximale Intensität hängt nur noch von der Güte des Vakuums ab. Bei dem hier beschriebenen Betatron (50 Hz!) müssen die Elektronen wegen ihres relativ kleinen Energiegewinnes pro Umlauf von etwa 14 bis $15 \mathrm{eV}$ eine erhebliche Strecke zurücklegen, bevor ihre Energie so groß ist, daß Stöße mit den restlichen Gasmolekülen genügend selten werden. Messungen mit dem Ionisationsmanometer zeigen, daß ein konstanter Dauerbetrieb ein Vakuum von ca. 5 bis $8 \cdot 10^{-6}$ Torr in der Röhre verlangt. Der weiter oben erwähnte zeitliche Intensitätsabfall von Glühkathoden läßt sich dagegen nicht durch Verschlechterung des Vakuums erklären, wie die zeitliche Verfolgung des Druckes in der Röhre während des Betriebes gezeigt hat.

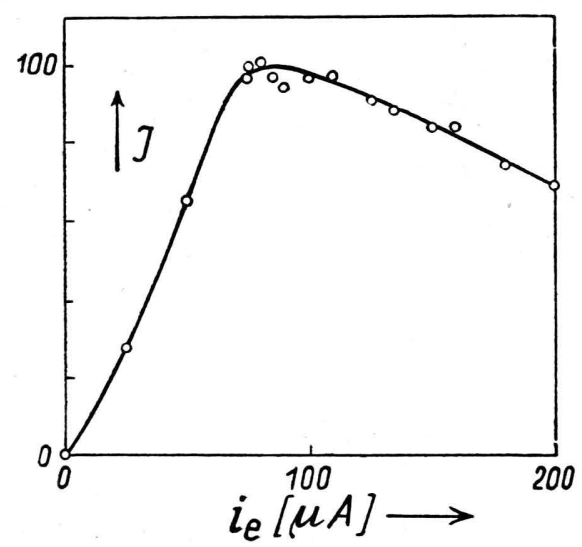

Abb. 10. Intensität $I$ der Röntgenstrahlen als Funktion des mittleren Elektronenstroms $i_{e}$ von der Elektronenquelle zur Ringröhre. 
Erreich te Intensität. Unter sorgfältiger Beachtung aller beschriebenen Einflüsse wurde mit der Apparatur eine reproduzierbare Maximalintensität der Röntgenstrahlung von $1,2 \mathrm{r} / \mathrm{min}$ im Dauerbetrieb erreicht. Wenn man diese Intensität mit der des 20-MV-Betatrons von $\mathrm{Kerst}^{6}$ ver. gleichen will, so sind vor allem die Betriebsfrequenz und die Einschußspannung zu berücksichtigen, während die Unterschiede in den übrigen Größen und Versuchsbedingungen eine geringere Rolle spielen. Die Frequenz hat bei Kerst den Wert $180 \mathrm{~Hz}$, das ist das 3,6-fache unserer Frequenz von $50 \mathrm{~Hz}$; die Einschußspannung beträgt bei Kerst $20 \mathrm{kV}$, also das 2,7-fache unseres Wertes $7,5 \mathrm{kV}$. Hiernach ist für das 20-MV-Betatron schon wegen dieser beiden Faktoren eine rd. 10fache Intensität zu erwarten. Experimentell erreichte Kerst mit seiner Apparatur $16 \mathrm{r} / \mathrm{min}$, d.h. rd. das 13-fache unserer Maximalintensität.

Die Betrachtung der Faktoren, welche die Intensität im wesentlichen beeinflussen, gibt gle:chzeitig auch die Richtung an, in der sich die Versuche zur Intensitätssteigerung beim 15-MV-Betatron bewegen müssen. Eine Erhöhung der Frequenz ist wünschenswert; sie läßt sich wohl ohne wesentlichen elektrotechnischen und materiellen Aufwand auf $150 \mathrm{~Hz}$ vergrößern. Die Einschußspannung sollte so hoch wie möglich gewählt werden; ohne größeren hochspannungstechnischen Aufwand lassen sich nach $\mathrm{Kerst}^{7}$ noch bis über $30 \mathrm{kV}$ an eine Kathode üblicher Bauart anlegen, wenn dies nur für genügend kleine Zeiten geschieht $\left(10^{-5}\right.$ bis $\left.10^{-6} \mathrm{sec}\right)$. Die kurze Einschußzeit hat gleichzeitig den Vorteil, daß der Einschießvorgang sich auf das Intervall beschränkt, innerhalb dessen die Elektronen wirklich „eingefangen“" werden können, und daß somit die Zahl der „falschen“ Elektronen herabgedrückt wird. Daß Verbesserungen der Elektronenquelle für die Intensitätssteigerung von Bedeutung sind, wurde bereits erwähnt.

\section{M essungen an der Röntgen-} strahlung

M e $B$ m e th od e n. Zum Nachweis der entstehenden Röntgenstrahlung diente ein Zählrohr üblicher Bauart, dessen Stöße auf einen Oszillographen gegeben wurden. Die Messung der Strahlungsintensität wurde mit einer Ionisationskammer ausgeführt, die von der

${ }^{6}$ D. W. Ke r st, Rev. sci. Instruments 13, 387 [1942].

${ }^{7}$ D. W. Ke r s t ${ }^{6}$, S. 391.
Firma C. H. F. Müller zur Verfügung gestellt wurde. Sie besteht aus einer Graphitkugel von $8 \mathrm{~cm}$ Durchmesser und $0,3 \mathrm{~cm}$ Wandstärke (Luftäquivalent). Die Kammer kann jederzeit mit Hilfe eines eingebauten Uranpräparates in $\mathrm{r} / \mathrm{sec}$ nachgeeicht werden durch Änderung der Spannungsempfindlichkeit des eingebauten Elektrometers. Die Übertragung dieses für Röntgenstrahlen bis etwa $1 \mathrm{MeV}$ üblichen Meßverfahrens auf die hier zu messenden Strahlen von wesentlich größerer Energie bringt an sich einen Meßfehler mit sich; eine Abschätzung zeigt aber, daß die Intensität nur um etwa $30 \%$ zu klein gemessen wird ${ }^{8}$; eine Korrektur der Meßwerte wurde nicht vorgenommen. Zeitweise wurde daneben ein integrierender Dosismesser nach Stra u \& („Mekapion“) mit einer Aluminiumkugel von $30 \mathrm{~cm}$ Durchmessèr und $0,5 \mathrm{~mm}$ Wandstärke verwendet, der von der Firma $R$ i c h a r d $\mathrm{S}$ e i f e r t u. Co. zur Verfügung gestellt wurde. Dieser Dosismesser ermöglichte eine bequeme Kontrolle der Tagesdosen für die im Labor tätigen Mitärbeiter (Länge eines Papierstreifens) und warnte durch Lichtsignale vor zu hoher Strahlendosis.

Energi e. Der überzeugendste Nachweis dafür, daß die projektierte Energie von $15 \mathrm{MeV}$ erreicht ist, wäre natürlich eine direkte Messung der $B \cdot r$-Werte für Elektronen, die nach dem Beschleunigungsvorgang die Ringröhre verlassen haben, oder eine direkte Bestimmung der maximalen Energie der Compton-Elektronen, die durch die austretenden Röntgenstrahlen ausgelöst werden. Beide Verfahren erfordern aber einen experimentellen Aufwand, für den uns die notwendigen Hilfsmittel nicht zur Verfügung standen. Die Energie wurde hier aus der Zeitdifferenz bestimmt, die zwischen dem Start der Elektronen und dem Augenblick der Entstehung der Röntgenstrahlung liegt. Diese Zeitdifferenz kann auf dem Oszillographenschirm als Länge abgegriffen werden, wenn man beide Zeitpunkte als Impulse auf die Wechselspannung schreibt, die der Induktionsfluß in einigen um den Kern oder um das Joch gelegten Windungen erzeugt. Der RöntgenstrahlenImpuls trat in der Regel bei einer Phase von $60^{\circ}$ bis $72^{\circ}$ auf, woraus mit Hilfe der gemessenen magnetischen Daten auf Maximalenergien von 14 bis $15 \mathrm{MeV}$ geschlossen werden kann.

Räumlich e Verteilung. Zunächst wurde die Verteilung der Röntgenstrahlung im gesamten Labor mit Hilfe der Ionisationskammer ausgemessen, um biologische Schädigungen der Mitarbeiter nach Möglichkeit zu vermeiden, da hier-

${ }^{8}$ Nach unveröffentlichten Rechnungen von $\mathrm{H}$. K u l e n k a m p ff. Wir danken Hrn. Prof. Kulenkampff für die ausführlichen Diskussionen in dieser Angelegenheit. 
für nicht nur die Intensität des Primärştrahls, sondern auch, die Streustrahlung von Bedeutung ist. Abb. 11 zeigt eine maßstäbliche Skizze des Labors und die Verteilung der Intensitätswerte, die im Bereich des Primärstrahls durch Linien gleichér Intensität dargestellt ist; die Intensität in Strahlmitte in einem Abstand von einem Meter von der Antikathode ist dabei gleich 1000 gesetzt. Der ausgezogene Pfeil gibt die Sollrichtung der Strahlmitte an. Die Intensität ist nicht symmetrisch dazu, was seinen Grund in der starken Streuwirkung des einen Eisenjoches hat, das von seitlichen Teilen des Primärstrahls getroffen wird. Auch die



Abb. 11. Intensitätsverteilung der Röntgenstrahlung im Labor.

Wand des Labors, in Abb.11 oben, liefert noch rückgestreute Strahlung. Bemerkenswert ist die starke Abschirmung der Strahlung durch das Eisenjoch, die dazu ausgenutzt werden kann, einen günstigen Platz für den an der Ionisationskammer Messenden zu finden. Die Strahlung des Betatrons nach links in Abb.11 ist gering und fällt mit der Entfernung rasch ab, so daß für die am Schalttisch Arbeitenden (linke Ecke unten) kein wesentliches Gefahrenmoment auch bei längerem Betrieb besteht.

In Abb. 12 sind einige Meßreihen wiedergegeben, die in $1 \mathrm{~m}$ Abstand von der Strahlenquelle senkrecht zum Strahl aufgenommen sind. In dieser Abbildung wird die Unsymmetrie durch die Streustrahlung besonders deutlich. Die Halbwertbreite, gewonnen durch Spiegelung der rechten
Kurvenhälfte an der Mittelachse (- - ), entspricht einem Strahlöffnungswinkel $2 \vartheta=40^{\circ}$. Vergleicht man diesen Wert unter Berücksichtigung



Abb. 12. Intensitätsverteilung im Röntgenstrahl.

der hier benutzten Dicke der Wolfram-Antikathode mit den Öffnungswinkeln, die von $\mathrm{K}$ u len kampff ${ }^{9}$ auf Grund der Theorie von Heitler ${ }^{10}$ berechnet sind, so erhält man für die ungefilterte Strahlung des Betatrons als Schwerpunkt der Energieverteilung (bezüglich des Streuwinkels) etwa $9 \mathrm{MeV}$. Messungen der Energieverteilung der entstehenden Röntgenstrahlung liegen bisher in diesem Energiebereich noch nicht vor; mit den theoretisch berechneten Kurven ist der angegebene Wert verträglich.

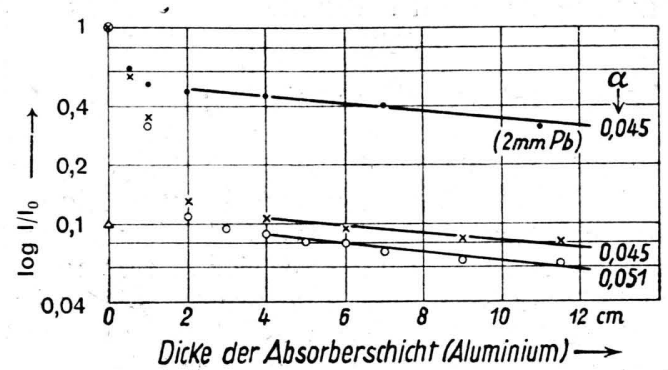

Abb. 13. Absorptionskurven der Röntgenstrahlen für Aluminium als Absorber mit den zugehörigen Werten des Absorptionskoeffizienten $\left(I / I_{0}\right.$ Verhältnis der jeweiligen Intensität zur Intensität ohne Absorber).

Absorption. Die Absorptionskoeffizienten vieler Metalle haben unterhalb $15 \mathrm{MeV}$ ein Minimum und befinden sich bei dieser Energie be-

${ }^{9} \mathrm{H} . \mathrm{K} \mathrm{u}$ le n k a m p f f, Ubberlegungen zur Bremsstrahlung energiereicher Ėlektronen, Jena, Febr. 1944 (unveröffentlicht).

10 W: Heitler, The Quantum Theory of Radiation, Oxford 1936. 
reits wieder im Ansteigen. Das gilt bekanntlich insbesondere für Blei. Wir wählten deshalb Aluminium, für das das Minimum des Absorptionskoeffizienten wenigstens erst an der oberen Grenze der hier in Betracht kommenden Strahlungsenergie liegt. Abb. 13 zeigt einige Meßreihen. Nach anfänglich sehr steilem Abfall, der wahrscheinlich Elektronen zuzuschreiben ist (die die Antikathode nicht getroffen und die Röhre direkt verlassen haben), läuft die Kurve in einen Ast aus, dessen Neigung sich für Absorptionskoeffizienten auswerten läßt. Als Mittelwert aus allen Messungen erhielten wir einen Absorptionskoeffizienten von $0,046 \mathrm{~cm}^{-1}$, ein Wert, der sich der von $\mathrm{He}$ i t l e r angegebenen Kurve in ihrem zwischen 10 und $25 \mathrm{MeV}$ gelegenen Minimum befriedigend einfügt.

\title{
Über den Elementarprozeß der Lichtanregung in Leuchtstoffen durch $\kappa$-Teilchen, schnelle Elektronen und $\boldsymbol{\gamma}$-Quanten II
}

\author{
Von Immanuel Broser und Hartmut Kallmann \\ (Z. Naturforschg. 2 a, 642-650 [1947]: eingegangen am 27. Juni 1947)
}

Aus dem Kaiser-Wilhelm-Institut für physikalische Chemie und Elektrochemie, Berlin-Dahlem

In Fortsetzung einer früheren Veröffentlichung werden eine Reihe von weiteren Versuchen an Leuchtstoffen mittels Sekundärelektronen-Vervielfacher-Photozelle als Meßgerät beschrieben. Mit einem Gleichstromverfahren werden $\mathrm{ZnS}, \mathrm{CaWO}_{4}, \mathrm{Zn}_{2} \mathrm{SiO}_{4}$, Naphthalin und einige andere Stoffe hinsichtlich ihrer relativen Lichtausbeute und Lichtdurchlässigkeit bei Anregung mit $\alpha$-Teilchen, schnellen Elektronen und $\gamma$-Strahlen untersucht. Die technische Lichtausbeute für $\alpha$-Teilchen ist beim $\mathrm{ZnS}$, für Elektronen und $\gamma$-Strahlen beim Naphthalin weitaus am besten. Es gelingt, neben einzelnen $\alpha$ Szintillationen im $\mathrm{ZnS}$ auch die Lichtwirkung einzelner Elektronen und $\gamma$-Quanten bei Benutzung von Naphthalin als'Leuchtstoff und unter Kühlung des Vervielfachers quantitativ nachzuweisen. Aus diesen am Elektronenstrahl-Oszillographen angestellten Beobachtungen werden Dauer und Intensität der Lichtimpulse bestimmt.

Tolgenden soll über die Fortsetzung unserer früher beschriebenen Versuche berichtet werden.

\section{Versuchsanordnung}

1. Es wurde mit der gleichen Versuchsanordnung wie in $I^{1}$ gearbeitet. Das emittierte Licht wurde mit einem sogenannten Elektronenvervielfacher gemessen, und zwar in Abhängigkeit von der Anregungsart und der Belegungsdichte (Masse pro $\mathrm{cm}^{2}$ ). Der benutzte Sekundär-Elektronenvervielfacher nach W e i ß war ein Vervielfacher mit 18 Netzen, der bei der verwendeter Spannung von ca. $2000 \mathrm{~V}$ einen Vervielfachungsfaktor zwischen $10^{5}$ und 106 ergab. Entsprechend der hohen Netzzahl war der Ruhestrom verhältnismäßig groß, etwa $10-8 \mathrm{~A}$. Nach genügend langer Einstelldauer war der Ruhestrom so konstant, daß bei Raumtemperatur Stromänderungen von der Größenordnung von $10^{-10}$ bis $10^{-11}$ A noch gemessen werden konnten. Das bedeutet, daß aus der primären photoelektrischen Schicht des Elektronenvervielfachers etwa 10-16 A austreten, also etwa 500 Elektronen pro Sekunde von der Photokathode abgegeben

1 I. B r o s e r u. H. K a $11 \mathrm{~m}$ a n n, Z. Naturforschg. 2a, 439 [1947]. werden müssen, um noch nachweisbar zu sein, oder, in Lichtquanten ausgedrückt, daß größenordnungsmäßig ein auffallender Lichtstrom von $10^{4}$ bis 105 Quanten pro Sekunde gerade noch nachweisbar ist, wenn man etwa an der Photoschicht mit einer Ausbeute 1 Elektron pro 100 Quanten rechnet.

Diese Anordnung war so empfindlich, daß mit ihr auch die Lichtemission von Stoffen festgestellt werden konnte, die im allgemeinen nicht mehr als Leucht- oder Fluoreszenzstoffe bezeichnet werden. So konnte z.B. die Lichtemission von Glas durch $\alpha$-Teilchen und Elektronen bei Verwendung eines radioaktiven Präparates, das nur etwa $10^{5}$ Teilchen pro sec emittierte, sowie durch $\gamma$-Strahlen noch ohne weiteres nachgewiesen werden. Bei diesen Stoffen wird nur ein sehr kleiner Bruchteil der an den untersuchten Stoff abgegebenen Energie in Iicht umgesetzt.

2. Die in Abschn.1 genannte Versuchsanordnung wurde wegen des großen Ruhestroms noch dahin modifiziert, daß nicht nur mit konstanter Belichtung gemessen wurde, sondern auch mit intermittierender Belichtung. Dann kann man die 